\title{
3D object-oriented image analysis in 3D geophysical modelling: Analysing the central part of the East African Rift System
}

\author{
I. Fadel ${ }^{a, b, *}$, M. van der Meijde ${ }^{a}$, N. Kerle ${ }^{a}$, N. Lauritsen ${ }^{c}$ \\ ${ }^{a}$ University of Twente, Faculty of Geo-information Science and Earth Observation (ITC), P.O. Box 6, 7500 AA Enschede, The Netherlands \\ b Geology Department, Faculty of Science, Helwan University, Ain Helwan, Egypt \\ c DTU Space, National Space Institute, Elektrovej, Building 327+328 and Ørsteds Plads, Building 348, DK-2800 Kgs. Lyngby, Denmark
}

\section{A R T I C L E I N F O}

\section{Article history:}

Received 15 August 2013

Accepted 8 November 2013

Available online 8 December 2013

\section{Keywords:}

Satellite gravity

$3 \mathrm{D}$ gravity model

Object-oriented image analysis

Inversion

Tanzania Craton

Seismic tomography

\begin{abstract}
A B S T R A C T
Non-uniqueness of satellite gravity interpretation has traditionally been reduced by using a priori information from seismic tomography models. This reduction in the non-uniqueness has been based on velocity-density conversion formulas or user interpretation of the 3D subsurface structures (objects) based on the seismic tomography models and then forward modelling these objects. However, this form of object-based approach has been done without a standardized methodology on how to extract the subsurface structures from the 3D models. In this research, a 3D object-oriented image analysis (3D OOA) approach was implemented to extract the 3D subsurface structures from geophysical data. The approach was applied on a 3D shear wave seismic tomography model of the central part of the East African Rift System. Subsequently, the extracted 3D objects from the tomography model were reconstructed in the 3D interactive modelling environment IGMAS+, and their density contrast values were calculated using an object-based inversion technique to calculate the forward signal of the objects and compare it with the measured satellite gravity. Thus, a new object-based approach was implemented to interpret and extract the 3D subsurface objects from 3D geophysical data. We also introduce a new approach to constrain the interpretation of the satellite gravity measurements that can be applied using any 3D geophysical model.
\end{abstract}

\section{Introduction}

Inverting gravity data has traditionally been one of the main geophysical challenges. Because of its monopole potential field, and resulting measured bulk property, retrieving accurate object information is often a matter of trade-offs and best guesses. Using $a$ priori geological and/or seismological data is a way of reducing this non-uniqueness of gravity inversions.

There are two main generic approaches have been followed in the past. The first is the conversion of the seismic velocities into densities using empirical standard velocity-density relationships (Birch, 1961; Gardner et al., 1974; Christensen and Mooney, 1995; Godfrey et al., 1997; Brocher, 2005) or including temperature, pressure, and mineral composition effects (Ravat et al., 1999; Korenaga et al., 2001; Cammarano et al., 2011). Calculated densities were then used to produce the estimated gravity signal and compare it with the measured gravity, or to constrain any further inversion process (Bezada and Zelt, 2011). This approach usually has to deal with

\footnotetext{
* Corresponding author.

E-mail addresses: i.e.a.m.fadel@utwente.nl, islam.geophysics@gmail.com (I. Fadel), m.vandermeijde@utwente.nl (M. van der Meijde),n.kerle@utwente.nl (N. Kerle), nlbla@space.dtu.dk (N. Lauritsen).
}

uncertainty in the seismic data and the velocity-density conversion relationships that result from the dependency on and uncertainty in physical parameters such as temperature, pressure and petrology (Bezada and Zelt, 2011; Cammarano et al., 2011). The second approach is based on the interpretation of the seismic models to derive objects (Götze and Lahmeyer, 1988) such as layers, plate boundaries, subduction zones, and domes. Subsequently the densities of these objects are calculated using one of the velocity-density relationships or through a trial and error approach. After that, the forward signal associated with these objects is compared with the measured gravity (e.g., Sanchez et al., 2011). A following step can be applying inversion approaches to reduce the misfit between the calculated and the measured gravity (e.g., Ebbing et al., 2001). This gravity modelling object-based approach has been done without a standardized methodology on how to extract the subsurface structures from 3D subsurface data, and has traditionally mainly been based on the user experience or pre-existing data (e.g., Ebbing et al., 2001; Sanchez et al., 2011).

In this research we report on a case study from the central part of the East African Rift System and how an advanced 3D objectoriented Image Analysis (3D OOA) approach can provide more objective definitions of object boundaries for gravity modelling and inversion. 3D OOA will be used to extract 3D objects from a tomography voxel model. Then, they will be reconstructed in the 3D 
object-based interactive gravity modelling environment IGMAS+. Subsequently their density contrast values will be calculated using an object-based inversion. Finally, the gravity response of the final model will be compared with the satellite gravity signal.

\section{Dataset}

\subsection{D tomography model}

The study area is the central part of the East African Rift System. Adams et al. (2012) developed a 3D shear wave seismic tomography model of the area, which is voxel-based and can be divided into two main sections. The first is the crustal part of the model, which extends from 0 to $40 \mathrm{~km}$ with $1 \mathrm{~km}$ increments. It represents the crustal structure of the model down to a flat Moho at $40 \mathrm{~km}$ depth. The second is the upper mantle part of the model ranges from a depth of $40 \mathrm{~km}$ down to $400 \mathrm{~km}$ at $10 \mathrm{~km}$ increments. This part of the model can be divided into two main zones, shallow and deep, based on their relative position in the model. The shallow zone of the upper mantle part (from $40 \mathrm{~km}$ down to $\sim 200 \mathrm{~km}$ ) is characterized by high velocities of the Tanzanian Craton and Ugandan basement complex, and low velocities of the surrounding tectonic settings such as rift branches, the Proterozoic mobile belts and Cenozoic volcanism. The deep zone of the upper mantle part (from $\sim 200 \mathrm{~km}$ down to $400 \mathrm{~km}$ ) is characterized by dominant low velocities related to a head plume or part of the deep mantle super plume in western Africa (Adams et al., 2012).

\subsection{Satellite gravity data}

The gravity data used in this study are GOCE only models (see van der Meijde et al. (2013) for details) and the high resolution EIGEN-6C (Förste et al., 2011), a combined gravity field model of LAGEOS/GRACE, GOCE and DTU10 up to a maximum degree and order of 1420 . The free air gravity data were obtained from the International Centre for Global Earth Models (ICGEM) web site (http://icgem.gfz-potsdam.de/ICGEM/) with a resolution of 0.1 degree (Fig. 1A). The effects of the nearby terrain were removed using ETOPO1 topography data (Fig. 1B), also downloaded through the ICGEM website with similar resolution ( 0.1 degree) to the models. The effect of the terrain correction on the satellite gravity measurements was small with a maximum value of $3.16 \times 10^{-5}$ $\mathrm{m} / \mathrm{s}^{2}$ (Fig. 1C), in line with findings by Mishra et al. (2012). Then, the data were Bouguer corrected, removing the effect due to the mass excess or deficiency as a result of high or low elevation relative to the mean sea level. The Bouguer correction also used ETOPO1 topography data with the same spatial resolution ( 0.1 degree) as the satellite gravity data and a reference density of $2.67 \mathrm{t} / \mathrm{m}^{3}$ (Fig. 1D).

\section{3D OOA}

Object oriented image analysis (OOA), also defined as object based image analysis (OBIA) or geographic object based image analysis (GEOBIA), is an image analysis technique that is based on the analysis of segments, or objects, instead of pixels. In general, OOA is a 2-step procedure starting with image segmentation that is followed by the classification of these segments. The segmentation step comprises of dividing image pixels into contiguous segments or objects, based on pre-defined knowledge based or homogeneity criteria, such as colour or intensity. The subsequent classification step uses the segments associated attributes which can be spectral, geometric, textural, contextual, and also geological and physical, to differentiate between the different objects present in the images. Consequently, OOA mimics cognitive visual image analysis techniques through a form of knowledge-driven analysis (Martha et al., 2011). Recently, 3D OOA was developed in the biomedical field to extract 3D objects from image stacks (Schoenmeyer et al., 2006). The first application to 3D synthetic and seismological models was recently carried out (Fadel et al., 2013). That study specifically applied the approach in eCognition software for deriving objects in a 3D seismic tomography model.

eCognition was the first OOA tool when introduced in 2000 and since then about $50-55 \%$ of all OOA published scientific studies have used it (Blaschke, 2010). Then the name was changed into Definiens in 2007. After that the package was split into two software packages; Definiens software that is related to biomedical applications, and eCognition that is mainly applied for geospatial applications. eCognition is built on the concept of rulesets that define the object extraction process in which a complex processing scheme of segmentation/classification algorithms can be implemented (Fadel et al., 2013). The ruleset is a semiautomatic since it is built on segmentation and classification algorithms, which are originally developed in the eCognition package, that are used to segment or to classify the 3D objects from the image stack. The user only needs to define the thresholds that are suitable to define the target segmentation scheme or the target object to be extracted.

3D OOA was applied on the 3D tomographic model in 2 stages. First, the ruleset of Fadel et al. (2013) was used to extract the objects for the upper mantle part of the model between $>40-400 \mathrm{~km}$ (Fig. 2 Upper Mantle Part). The upper mantle objects can be separated in two zones based on their relative depth in the upper mantle part of the model:

(1) The shallow zone $(>40-\sim 200 \mathrm{~km})$ consists of high velocity objects (craton (C) and shallow high velocity object (SHV)), low velocity object (rifts (R)), and boundary zones surrounding both high and low velocity objects (boundary shallow high velocity (BSHV) and a boundary shallow low velocity (BSLV), respectively).

(2) The deep zone $(\sim 200-400 \mathrm{~km})$ is dominated by a low velocity object (LV) with its inner part (ILV) followed by a high velocity object at the deepest part of the model (deep high velocity (DHV)) with a boundary between it and the upper low velocity object (boundary deep low velocity (BDLV)).

Second, a new ruleset was developed for the crustal part of the model from the surface down to a depth of $40 \mathrm{~km}$. The new crustal part of the model (Aubreya Adams, personal communication, April 29,2013 ) was voxel based with $1 \mathrm{~km}$ depth interval.

The new ruleset for this part was based on visual interpretation and analysis of the 3D histogram, summarizing the 3D distribution of density values with depth (Fig. 3B), similar to Fadel et al. (2013). The tomographic model had a hard boundary of 2 discrete $1 \mathrm{~km}$ layers above and below the Moho (Adams et al., 2012), with a major velocity jump over these two layers. The ruleset made use of this hard boundary to distinguish the crustal part from the upper mantle part. The new ruleset consisted of 2 main steps (see supplementary data for more details).

In the first step multi-threshold segmentation was used to separate the crustal from the upper mantle part of the model. This algorithm splits the image into segments and classifies them based on pre-defined thresholds. $40 \mathrm{~km}$ depth was used as a threshold to separate the crustal from the upper mantle part (Fig. 3A). The subsequent focus was on the crustal part of the model ( $0-40 \mathrm{~km})$. A series of assign class algorithms was applied to split it into five objects using thresholds that were defined based on the visual interpretation supported by the analysis of the 3D histogram (Fig. 3B). These 5 units (Fig. 2 Crustal Part) do not necessarily have a geo/physical meaning in terms of their velocities, due to the sharp boundary with large velocity contrast at the fixed layer at $40 \mathrm{~km}$ depth in the tomography model. However, they highlight the relative velocity changes in this zone. Unit 1 was characterized by very low velocity 

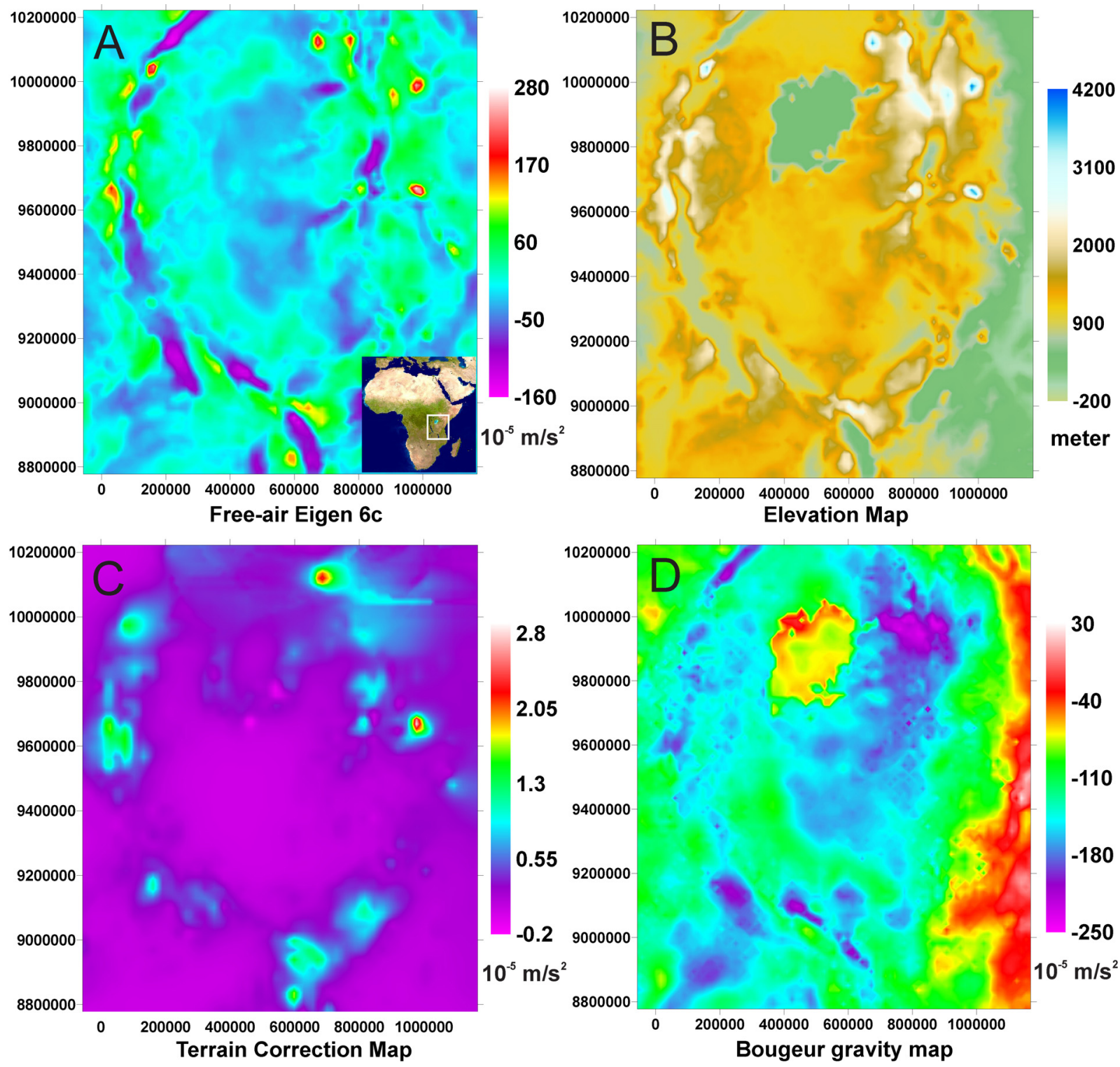

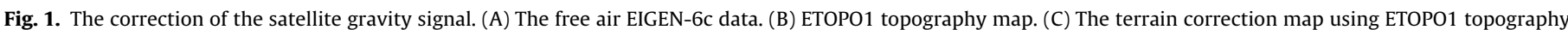
and reference density $2.67 \mathrm{t} / \mathrm{m}^{3}$. (D) The Bouguer anomaly map after the Bouguer and terrain corrections.

values up to $3.2 \mathrm{~km} / \mathrm{s}$, and was surrounded by unit 2 which represents the boundary for unit 1 and has a velocity range from $3.2 \mathrm{~km} / \mathrm{s}$ up to $3.4 \mathrm{~km} / \mathrm{s}$. Unit 3, with a velocity range of $3.4-3.55 \mathrm{~km} / \mathrm{s}$, was characterized by low variation in the density distribution as can be seen in the 3D histogram. Unit 4, with a velocity range of $3.55-4.1 \mathrm{~km} / \mathrm{s}$, surrounded the final unit 5 that was characterized by velocities higher than $4.1 \mathrm{~km} / \mathrm{s}$ thereby representing the highest velocity in the crustal part. 3D OOA behaved as any simple isosurface due to the lack of $a$ priori information about the crustal part; in addition to the limited ability of seismic tomography techniques in such shallow depths where other approaches are more preferable such as receiver functions (Liu and $\mathrm{Gu}, 2012$ ).

\section{Forward modelling}

\subsection{Objects reconstruction}

The objects extracted in the OOA part were re-constructed in IGMAS+ (3D Interactive Gravity and Magnetic Application System; Götze and Lahmeyer, 1988; Schmidt et al., 2010), which facilitates interactive forward modelling of the subsurface in an object-oriented environment. The results from OOA were exported as classified cross sections that were used to constrain the shape of the objects through the reconstruction process. 27 sections were created in IGMAS+ with $50 \mathrm{~km}$ average distance between them to fit the original 3D tomography model that had a horizontal resolution of 0.5 degree. Then, OOA classified sections were imported in IGMAS+ and co-located with the created IGMAS+ sections to start re-constructing the objects. The upper mantle part of the model (>40-400 km depth) was constructed first (see Fig. 4 Upper Mantle Part and Fadel et al. (2013) for details). Adams et al. (2012) used a $1 \mathrm{~km}$ thick layer above and below the $40 \mathrm{~km}$ depth to constrain Moho depths and allow for large variations in the velocity between the crustal and the upper mantle part of the model. Hence, a $1 \mathrm{~km}$ horizontal layer above and below a depth of $40 \mathrm{~km}$ ( $2 \mathrm{~km}$ thick layer between depths $39-41 \mathrm{~km}$ ) was constructed to represent the Moho as in the original model. This is in line with actual observations on the Moho which often indicate it is a layer with a certain thickness and not a discrete boundary. Subsequently, the crustal part of the model was constructed above (Fig. 4 Crustal Part).

The tomographic model did not have a realistic Moho topography. Therefore, another model was constructed in which the 


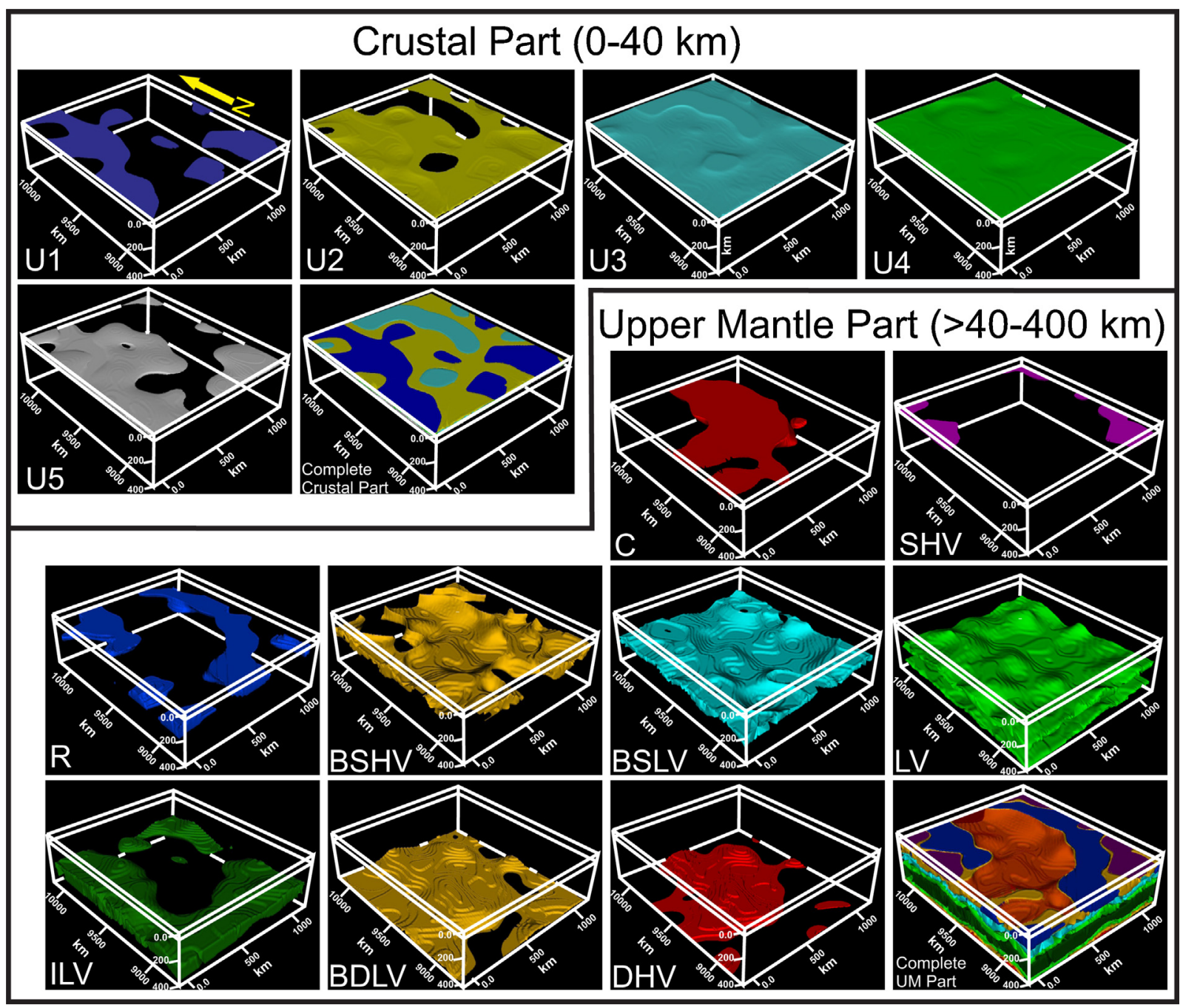

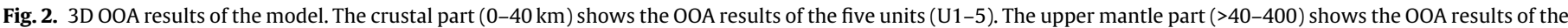

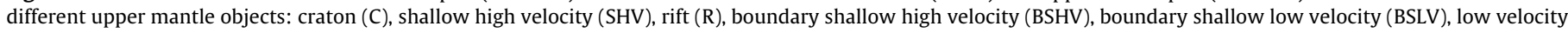

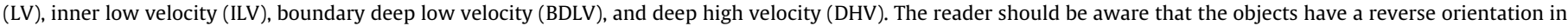
the East-West direction (compare them with Fig. 4) which is a visualization defect of 3D OOA results.

Adapted from Fadel et al. (2015).

crustal and the upper mantle parts remained the same; however, the fixed Moho was replaced with a gravity based crustal thickness map of Africa (Tugume et al., 2013). The $2 \mathrm{~km}$ thick flat layer representing top and bottom of the Moho transition zone was modified to describe the undulation of the new Moho. Now a realistic Moho topography was included, representing spatial density variations due to Moho undulations. The embedded Moho model showed a large variation in crustal thicknesses that ranged from $\sim 28 \mathrm{~km}$ at coastal regions to $\sim 42 \mathrm{~km}$ in the central part of the study area (Fig. 5). The alternative option to use crustal thickness from CRUST2.0, a crustal thickness map that was created by interpolating the crustal velocity estimates from active-source profiling techniques and geologically estimated terrain ages (Bassin et al., 2000), was not considered because of the debate of using CRUST2.0 in data poor environments (see discussion in van der Meijde et al., 2013).

\subsection{Object-based inversion}

After the definition of the two scenario models based on the results of the 3D OOA, density values for each object were needed to calculate the gravity signal and compare it with the measured satellite signal. Schmidt et al. (2011) introduced a linear objectbased inversion approach in IGMAS+, based on the minimum mean square error (MMSE) algorithm introduced by Haase (2008) based on the work of Saether (1997). The interactive object-based modelling approach aims to minimize the misfit between the calculated and the measured gravity signal up to approximation error:

Calculated $=$ measured - error $=\sum_{i=1}^{n} \rho_{i} P_{i}$

where $n$ is the total number of bodies, $\rho_{i}$ is the constant density of body $i$, and $P_{i}$ represents the polyhedron gravity effect of body $i$ (for density 1$)$.

Eq. (1) simplifies the subsurface structure into a small number of objects. Each object has a constant density value $\left(\rho_{i}\right)$ that is the common value of all voxels within this object $i$. The calculated gravity effect of object $i$ will be the product of its density $\rho_{i}$ multiplied by the polyhedron gravity effect in case of a unit density value (e.g., $1 \mathrm{t} / \mathrm{m}^{3}$ or $1 \mathrm{~kg} / \mathrm{m}^{3}$ or any other unit). The total calculated gravity signal will be the summation of the resulting polyhedron effects for the total number of objects $n$. The object-based inversion approach aims to find the optimal density values $\rho_{i}$ using MMSE estimation 

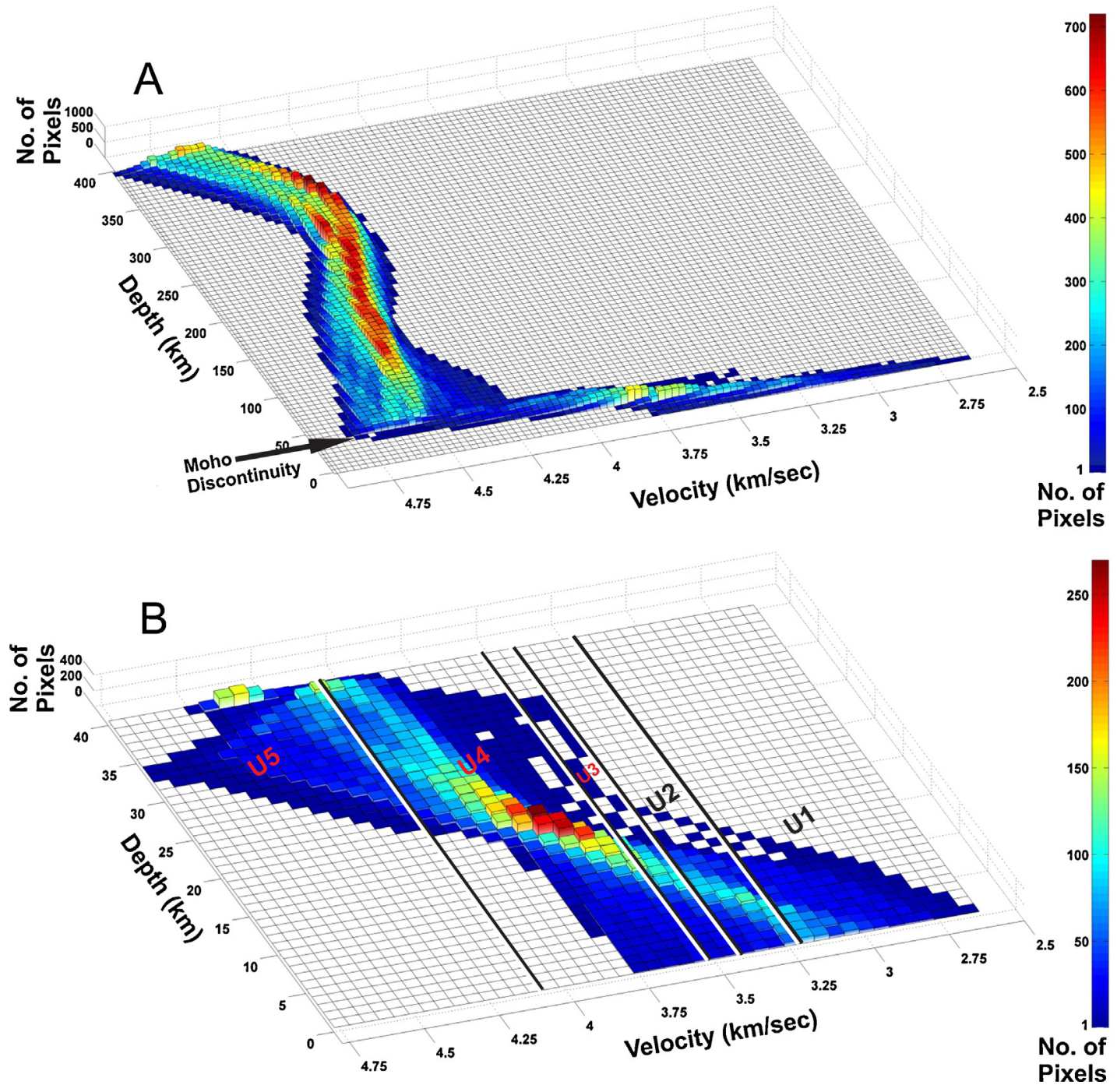

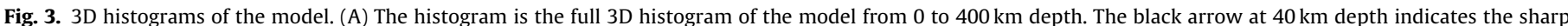

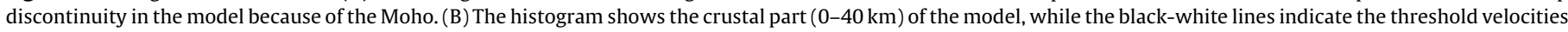
for the different units.

instead of the traditional interactive trial and error approaches. The estimated optimized density values $\rho_{i}$, directly related to the defined objects produce a calculated gravity signal with a minimum misfit (error) to the measured signal. It is also possible to add constraints to the inversion process in case of the presence of $a$ priori knowledge about the density values of some objects in the model. In that case, the objects with a priori known density values are provided in the inversion algorithm as constants while the inversion algorithm is only applied to the objects with unknown density values, as shown in Eqs. (2) and (3).

Calculated $=\sum_{i p} \rho_{i p} P_{i p}+\sum_{n p} \rho_{n p} P_{n p}$

Hence by substitution using Eq. (1),

Measured - error $-\sum_{n p} \rho_{n p} P_{n p}=\sum_{i p} \rho_{i p} P_{i p}$

where index ip indicates the objects that will be inverted, and $n p$ indicates the objects that will not. A point of attention is the setting of the background density value. The Bouguer anomaly is the result of the density contrast between the background densities of the Earth and the existing densities of the different objects in the subsurface. IGMAS+ allows one density background value to calculate density anomalies and, hence, gravity anomalies relative to it. However, for models with large vertical and spatial density contrasts down to $400 \mathrm{~km}$ the background density values changes with depth. Therefore, the use of one value as a density background is not ideal. In order to deal with this limitation, relative object density contrasts were used with respect to a background density of 0 . The results from the inversion process are thus density contrast values between objects in the model and the background densities at their equivalent depths. For example, if we assumed that the density background for $0-10 \mathrm{~km}$ is $2.67 \mathrm{t} / \mathrm{m}^{3}$, the absolute density value of unit 1 is $2.453 \mathrm{t} / \mathrm{m}^{3}$ (U1 lies in a depth range $0-10 \mathrm{~km}$ with a density contrast of -0.217 , as shown in Table 2 ). The measured gravity signal was filtered to remove short wavelengths in order to fit the resolution of the input data (i.e., the seismological model). The smallest object in the model is approximately $100 \mathrm{~km}$. This required the application of a low-pass cosine roll-off filter on the measured gravity signal (Fig. 6D). This removed the high frequency content, needed to be comparable with the calculated gravity signal based on the seismological model. The filtered gravity signal was used to estimate the optimized density contrast values of the different objects, and to evaluate the degree of correlation with the calculated gravity signal. 

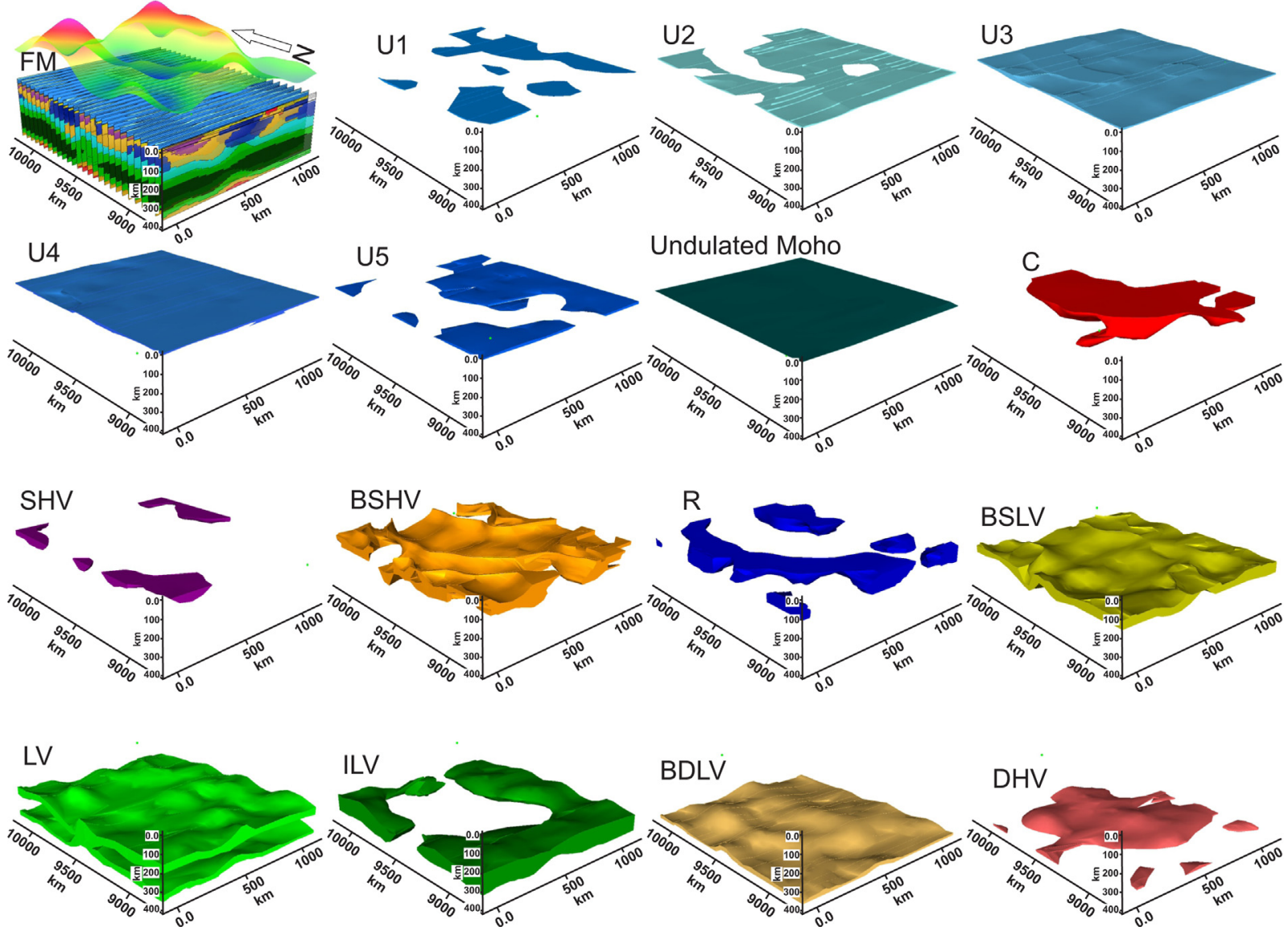

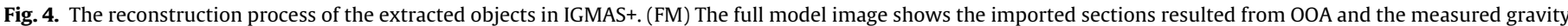

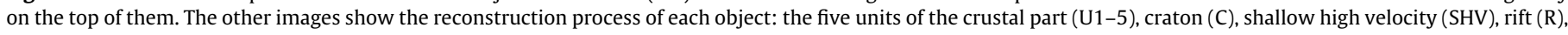

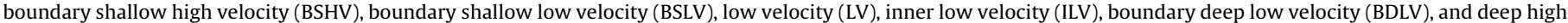
velocity (DHV).

Table 1 shows the results of the inversion process for the different parts of the two models, with both the flat and the undulated Moho, compared to EIGEN-6c. In both cases, the calculated gravity signal from the upper mantle part showed 69\% correlation with the measured satellite gravity. In case of the flat Moho model, the added Moho discontinuity layer and the crustal part only had a slight effect on improving the results (maximum of $3 \%$ improvement). In case of the complete model, however, using the realistic Moho surface based on the crustal thickness map of Africa (Tugume et al., 2013), the results showed a major improvement up to a 95\% fit. The undulating Moho discontinuity clearly has a strong influence on the final fit, which is to be expected for a large-scale strong anomaly that also has a significant contribution to the gravity signal among all modelled objects. Another reason for the increased fit is that the applied Moho topography is also derived from gravity data. Therefore, it is expected to give a high correlation and consequently contribute strongly. This strong contribution is reflected in the, unrealistically, high relative density contrast value $5.157 \mathrm{t} / \mathrm{m}^{3}$ of the Moho layer that results from the inversion, as shown in Table 2. This is also partly because the Moho is modelled as a thin layer ( $2 \mathrm{~km}$ thick) and that all the uncertainties in the strong negative crustal density values (U1 $-0.217 \mathrm{t} / \mathrm{m}^{3}$, U2 $-0.244 \mathrm{t} / \mathrm{m}^{3}$, U3 $-0.278 \mathrm{t} / \mathrm{m}^{3}$, U4 $-0.312 \mathrm{t} / \mathrm{m}^{3}$, and $\mathrm{U} 5-0.373 \mathrm{t} / \mathrm{m}^{3}$, Table 2) seem to accumulate in the high positive density contrast value of the Moho.

Table 2 (second column) shows the density contrast values for each individual object in the model resulting from the object-based inversion using EIGEN-6c. The values for the crustal part (U1-U5) of the model, rifts (R), the low velocity zone (LV), and its inner part (ILV) showed negative density contrast. For the rifts (R), the low velocity zone (LV), and the inner low velocity zone (ILV), the values are matching with the low velocity values from the tomography model. However, the inner low velocity (ILV) object showed

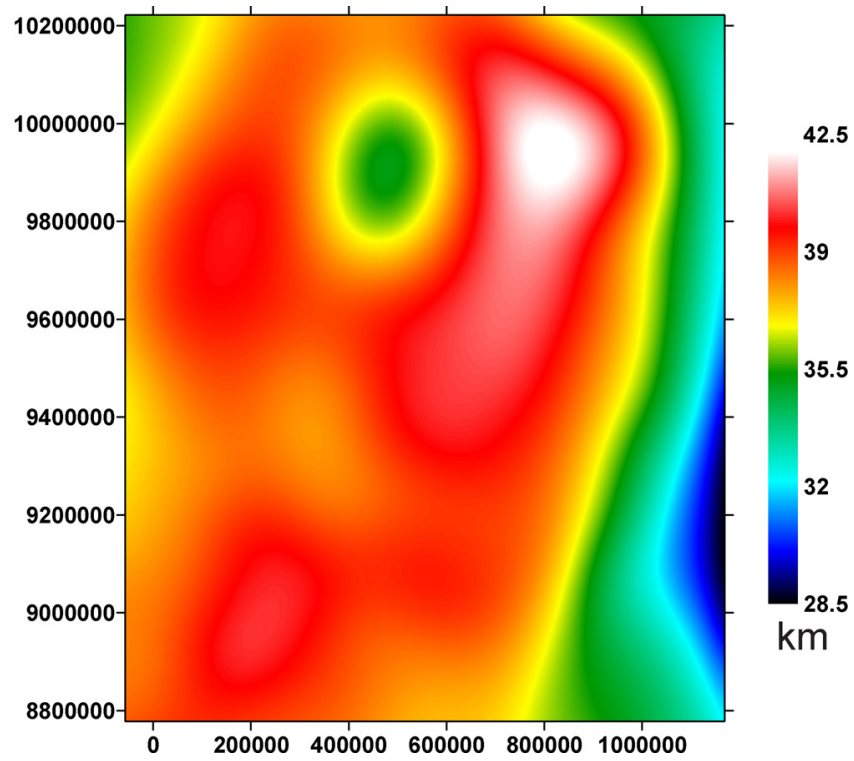

Fig. 5. The crustal thickness map of the study area adapted from Tugume et al. (2013). 

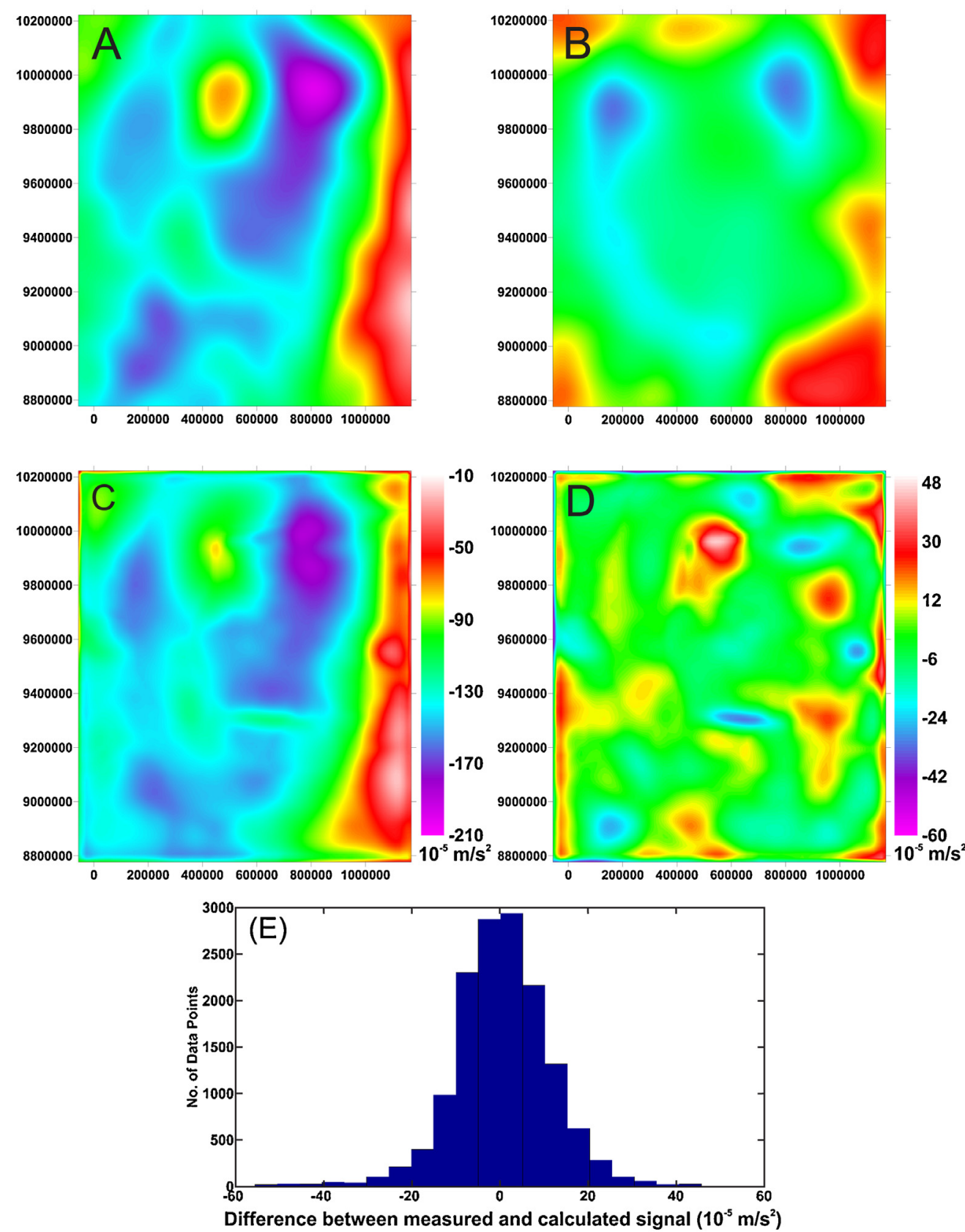

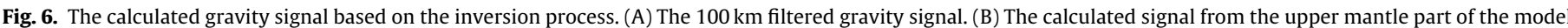

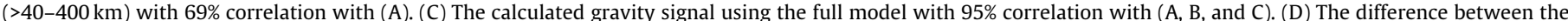
measured $(A)$ and the calculated $(C)$ signal. (E) The histogram of difference map (D).

less negative density contrast than the low velocity (LV) object, which contradicts the seismic tomography model, since the inner low velocity (ILV) had the lowest velocity. The craton (C), the shallow high velocity (SHV), and the deep high velocity zone (DHV) objects, showed positive density contrast values that agreed with the high velocity in the tomographic model. However, the craton (C) had a higher velocity in the tomographic model than the shallow high velocity (SHV) object. This was contrary to the density

Table 1

The inversion results for the different parts of the two models.

\begin{tabular}{|c|c|c|c|c|}
\hline Parameters & Upper mantle part & Upper mantle part with Moho & Complete model & Complete model without Moho \\
\hline \multicolumn{5}{|l|}{ Flat Moho model } \\
\hline Correlation & 0.69 & 0.71 & 0.72 & 0.70 \\
\hline Standard deviation $\times 10^{-5} \mathrm{~m} / \mathrm{s}^{-2}$ & 25 & 24 & 14 & 25 \\
\hline \multicolumn{5}{|l|}{ Undulated Moho model } \\
\hline Correlation & 0.69 & 0.71 & 0.95 & 0.89 \\
\hline Standard deviation $\times 10^{-5} \mathrm{~m} / \mathrm{s}^{-2}$ & 25 & 24 & 11 & 16 \\
\hline
\end{tabular}


Table 2

The density contrast $\left(\mathrm{t} / \mathrm{m}^{3}\right)$ estimated from the inversion of the complete model with the undulated Moho using different gravity models.

\begin{tabular}{|c|c|c|c|c|c|}
\hline Object & Eigen- $6 c\left(t / m^{3}\right)$ & Eigen- $6 c 2\left(t / m^{3}\right)$ & $\operatorname{GOCO03S}\left(\mathrm{t} / \mathrm{m}^{3}\right)$ & GO-CONS-GCF-2-DIR-R4 (t/m³) & GO-CONS-GCF-2-TIM-R4 ( $\left.\mathrm{t} / \mathrm{m}^{3}\right)$ \\
\hline Unit 1 (U1) & -0.217 & -0.215 & -0.211 & -0.211 & -0.211 \\
\hline Unit 2 (U2) & -0.244 & -0.243 & -0.24 & -0.241 & -0.241 \\
\hline Unit 3 (U3) & -0.278 & -0.277 & -0.275 & -0.275 & -0.275 \\
\hline Unit 4 (U4) & -0.312 & -0.312 & -0.308 & -0.309 & -0.308 \\
\hline Unit 5 (U5) & -0.373 & -0.374 & -0.371 & -0.372 & -0.371 \\
\hline Craton $(\mathrm{C})$ & 0.008 & 0.008 & 0.007 & 0.007 & 0.007 \\
\hline Shallow High Velocity (SHV) & 0.011 & 0.011 & 0.01 & 0.01 & 0.009 \\
\hline Boundary Shallow High Velocity (BSHV) & 0.005 & 0.005 & 0.004 & 0.004 & 0.004 \\
\hline $\operatorname{Rift}(\mathrm{R})$ & -0.002 & -0.002 & -0.003 & -0.003 & -0.003 \\
\hline Boundary Shallow Low Velocity (BSLV) & -0.015 & -0.015 & -0.014 & -0.014 & -0.014 \\
\hline Low Velocity Zone (LV) & -0.052 & -0.053 & -0.053 & -0.053 & -0.053 \\
\hline Inner Low Velocity (ILV) & -0.002 & -0.002 & -0.003 & -0.003 & -0.003 \\
\hline Boundary Deep Low Velocity (BDLV) & -0.067 & -0.067 & -0.062 & -0.06 & -0.058 \\
\hline Deep High Velocity (DHV) & 0.172 & 0.173 & 0.171 & 0.172 & 0.17 \\
\hline MOHO & 5.157 & 5.154 & 5.132 & 5.137 & 5.139 \\
\hline Reference & 0 & 0 & 0 & 0 & 0 \\
\hline Correlation & 0.948 & 0.949 & 0.948 & 0.948 & 0.947 \\
\hline Standard deviation & 11.021 & 11 & 11.002 & 11.062 & 11.089 \\
\hline
\end{tabular}

contrast, which was lower for the craton. This contradiction can be explained by the large uncertainty in the velocity values that is less than $0.2 \mathrm{~km} / \mathrm{s}$ at depths $<250 \mathrm{~km}$, and not more than $0.3 \mathrm{~km} / \mathrm{s}$ at greater depths (Adams et al., 2012). The boundaries between Shallow High Velocity (BSHV), Shallow Low Velocity (BSLV), and Deep Low Velocity (BDLV) objects showed realistic transition values. The final maps of the calculated gravity signal for the upper mantle part and the complete model with the undulated Moho are shown in Fig. 6.

\subsection{Testing different gravity models}

The previous modelling was done using a combined EIGEN$6 c$ model constructed from satellite data and other gravity data sources. This raised the question of how much the different content, resolution and quality of different gravity models influence the final model parameters. For this purpose, different combined and satellite data only models were compared. EIGEN-6c, EIGEN$6 \mathrm{c} 2$ (GOCE, GRACE, LAGEOS and surface data), GOCO03S (GOCE, GRACE, CHAMP, SLR), GO-CONS-GCF-2-DIR-R4 (GOCE, GRACE, and LAGEOS), and GO-CONS-GCF-2-TIM-R4 (GOCE only) were used in this test (for more information on the GOCE related models see van der Meijde et al. (2015)). The models were downloaded with the same resolution and subjected to the same processing (terrain correction, Bouguer correction and $100 \mathrm{~km}$ cosine roll-off filter). The results for the different gravity models are summarized in Table 2.

The results showed that EIGEN-6c2, the most recent and highest resolution model, gives the best results. This was expected since the model has been shown to have a higher accuracy than any other gravity model (Förste et al., 2013). However, the differences in the models (Fig. 7) were not significant. In addition, the correlations are all high and comparable, and standard deviation results vary little (Table 2). When compared to the standard deviation that is obtained for the best model (see Table 1) one can conclude that choices made in the creation of the model are of much greater consequence than the variability between models (at $100 \mathrm{~km}$ resolution).

\section{Discussion}

It is difficult to quantify how much 3D OOA has improved the modelling of subsurface objects. An expert modeler might have come to a similar result, or maybe even better. The main advantage is that the boundaries are based on physical rules and knowledge, and that they are objective and repeatable. An additional advantage was that the top and bottom of the objects were determined, something that traditionally has been difficult due to strong smearing and/or smoothing in the vertical direction.

In this example the boundaries of the objects were fixed, and the inversion only influenced the internal physical properties of the objects. This is, of course, a limitation and can be given more degrees of freedom in future attempts. The method we developed is ideal for incorporating a priori information in the identification of objects. We only used geological knowledge on the main geological and tectonic features in the area based on Adams et al. (2012), and crustal thickness from Tugume et al. (2013). More a priori information can potentially be incorporated, such as velocity-density relationships, point observations from, for example, receiver function analysis, and petrochemical knowledge on the physical properties of the various defined objects. This way physical properties can be further constrained. In our experiments we observed that for some object parameters had non-realistic density contrast values (e.g., the Moho layer). By adding ranges within which parameters may vary, the result is likely to be more realistic in terms of retrieved physical properties.

Because of the poorly resolved crustal part of the seismological model (Adams et al., 2012), there is no strictly defined structural integrity between the crustal and the upper mantle part of the model. The sharp Moho boundary defined in the tomographic model is not physically realistic in all places, and replacement by another crustal thickness model creates an uncertainty in the physical continuation of the physical properties, thereby adding more uncertainty on structural integrity.

The $31 \%$ misfit value between the calculated and measured gravity, based on the upper mantle part of the model, can be explained due to the absence of the crustal part in the model. The dramatic improvement in the correlation and standard deviation after adding the Moho are doubtful when considering the unrealistically high density contrast of the Moho that had a high influence on the measured signal. Therefore, in order to prove the necessity to add a realistic Moho surface, the inversion was carried out excluding the Moho layer (a density contrast $=0$ ) from the inversion using $n p$ index mentioned in Eqs. (2) and (3). The results of the inversion showed a significant improvement of $89 \%$ in the calculated signal (Table 1). This underscores the importance of knowledge on the shape of the Moho surface. In addition, it reflects the necessity for density contrast constraints of some objects, since a minor change in one object in the model caused significant changes in the density contrast of all objects. Table 2 shows that a negligible change in the measured gravity signal can cause substantial changes in the 

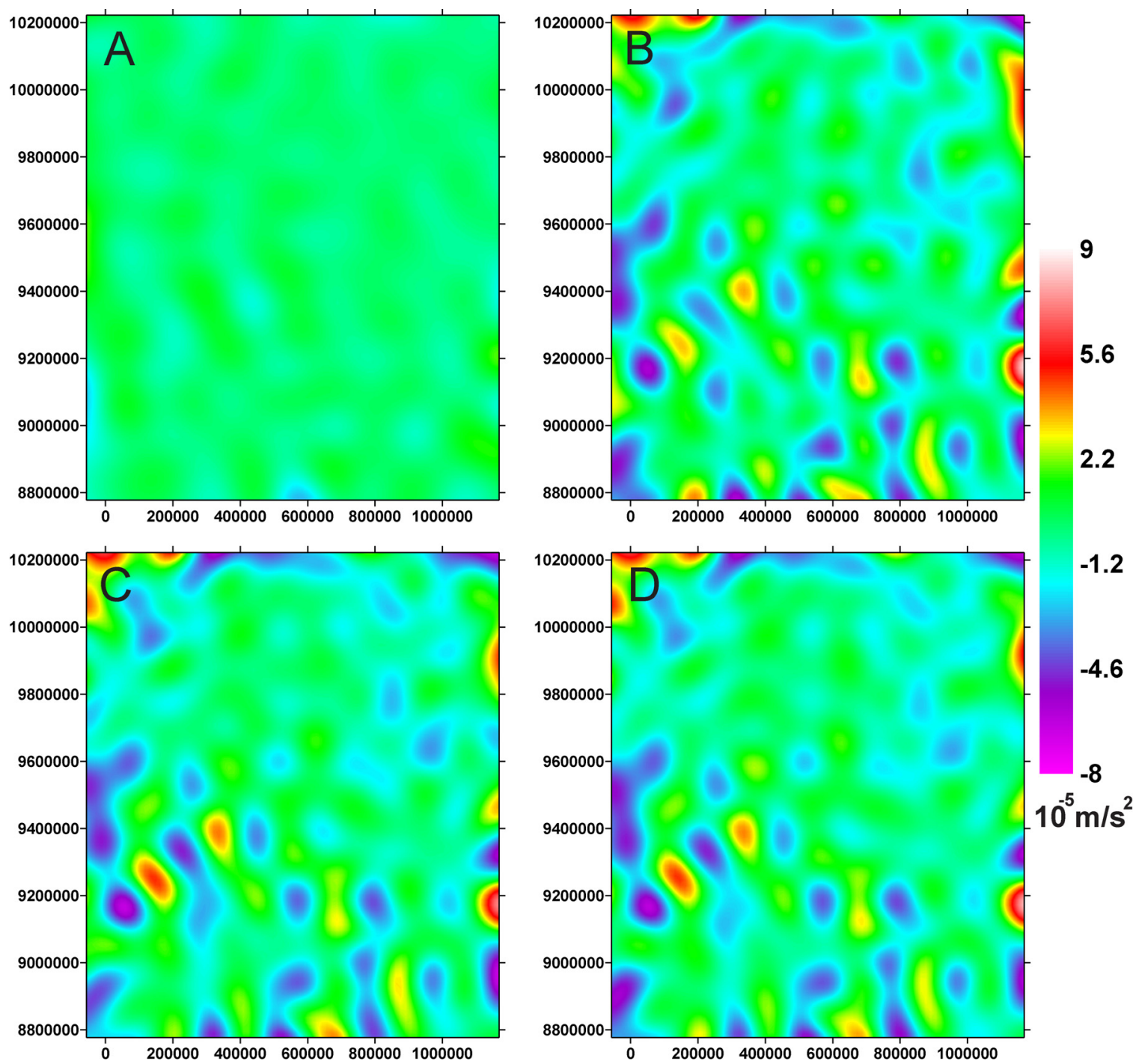

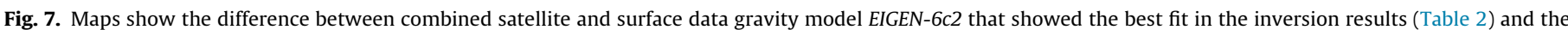

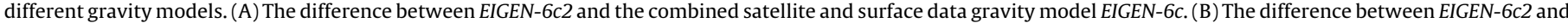

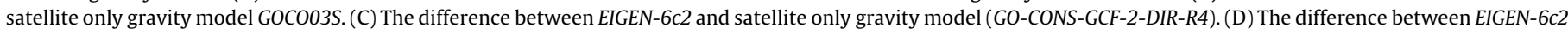
and satellite only gravity model (GO-CONS-GCF-2-TIM-R4).

density contrast values. Another indicator for the high sensitivity of the density contrast values is that the values are not stable with changes in the extent of the measured gravity signal. Some tests were done to remove the edge effect in the calculated gravity signal by reducing the measured gravity extent by 4,8 , and $12 \%$ of the total extent of the study area. The results of the density contrast for each trial were also different. Moreover, the edge effect was still present in the forward signal and the misfit between the measured and the calculated gravity signal (Fig. 6C and D) since the forward calculation of IGMAS+ is based on flat Earth assumptions and does not take in to account the spherical shape of the Earth.

The uncertainty in the location of the extracted objects are related to the uncertainty of the seismic tomographic model. Adams et al. (2012) stated that the uncertainties for major features in the model are ranging from 25 to $50 \mathrm{~km}$ for the shallow zone of the upper mantle part of the model $(40-250 \mathrm{~km})$ and can be up to $75-100 \mathrm{~km}$ for depths $>250 \mathrm{~km}$. The uncertainty of the crustal part of the model (0-40 km) was not discussed in Adams et al. (2012) but was assumed to be the same as for the depth range $40-250 \mathrm{~km}$.

The uncertainties are reflected in the results of the object based inversion. The correlation factor and the standard deviation of the inversion results show that for the complete model, including the undulated Moho density contrasts $5.157 \mathrm{t} / \mathrm{m}^{3}$, the correlation was 0.95 and the standard deviation of the misfit was around $11 \times 10^{-5} \mathrm{~m} / \mathrm{s}^{2}$ (Fig. $6 \mathrm{D}$ and $\mathrm{E}$ ). The misfit can be explained by the inherent uncertainty in the seismic model that also include the object boundaries. The inversion algorithm only allowed change in the density contrasts. That resulted in some objects density contrast to be unrealistic. However, if the algorithm was allowed to change the boundaries location within certain limits that are linked to the uncertainty of the input data, may be both the location of the objects' boundaries and the density contrast values will be improved. This can be done through implementing insight on a priori knowledge about the possible density contrast ranges of the objects that can prevent adding more non-uniqueness to the problem due to the available infinite combination of boundary locations and density contrast values.

\section{Conclusion}

In this research we developed a method to extract 3D objects from a seismic tomography model using 3D OOA. The extracted 
objects were then reconstructed in an object-based gravity modelling environment (IGMAS+), and the density contrast value for each object calculated using an object-based inversion technique. The calculated gravity signal from the upper mantle part $(>40-400 \mathrm{~km})$ of the model produced a $69 \%$ correlation with observed gravity. The low fit is mainly due to the absence of the Moho undulation surface, and the crustal part. By adding a flat Moho layer with a $2 \mathrm{~km}$ thickness and the crustal objects, based on the tomographic model, the calculated gravity signal only improved by $3 \%$. However, by adding the Moho undulation surface based on the crustal thickness map of Africa by Tugume et al. (2013) and, the intra-crustal objects from the tomographic model from Adams et al. (2012), the fit of the calculated gravity signal with the observed gravity significantly improved up to $95 \%$. However, the results for the Moho and the crustal part need further verification, and further improvements are expected based on other geophysical data sources, such as receiver functions.

The usage of the different satellite gravity models, either satellite only models or satellite and terrestrial models, only had a minor effect on the inversion results. Uncertainty in the inversion is larger than the variability due to the use of different models (all at $100 \mathrm{~km}$ resolution).

Integrating 3D OOA in a 3D object-oriented gravity modelling environment can lead to a significant improvement in converting the voxel-based geophysical models into object-based models based on simple and objective knowledge-based classification rules. In addition, the object-based inversion approach can be improved by using the different available segmentation algorithms, which, for example, allow objects to grow and shrink automatically, and hence allow some flexibility for automatic adjustment of the boundary location, and thus to optimize fitting results.

\section{Acknowledgments}

We thank Dr. Aubreya Adams at Washington University for making the seismic tomography model of the study area available for this study. We would like to thank Prof. Dr. Hans-Jürgen Götze and his research team at Kiel University for providing the academic license for the IGMAS+ software.

\section{Appendix A. Supplementary data}

Supplementary data associated with this article can be found, in the online version, at http://dx.doi.org/10.1016/j.jag.2013.11.004.

\section{References}

Adams, A., Nyblade, A., Weeraratne, D., 2012. Upper mantle shear wave velocity structure beneath the East African plateau: evidence for a deep, plateauwide low velocity anomaly. Geophysical Journal International 189 (1), 123-142.

Bassin, C., Laske, G., Masters, G., 2000. The current limits of resolution for surface wave tomography in North America. EOS Transactions AGU 81, F897.

Bezada, M.J., Zelt, C.A., 2011. Gravity inversion using seismically derived crustal density models and genetic algorithms: an application to the Caribbean-South American plate boundary. Geophysical Journal International 185 (2), 577-592.

Birch, F., 1961. The velocity of compressional waves in rocks to 10 kilobars: 2. Journal of Geophysical Research 66 (7), 2199-2224.

Blaschke, T., 2010. Object based image analysis for remote sensing. ISPRS Journal of Photogrammetry and Remote Sensing 65 (1), 2-16.
Brocher, T., 2005. Empirical relations between elastic wave speeds and density in the Earth's crust. Bulletin of the Seismological Society of America 95 (6), 2081-2092.

Cammarano, F., Tackley, P., Boschi, L., 2011. Seismic, petrological and geodynamical constraints on thermal and compositional structure of the upper mantle: global thermochemical models. Geophysical Journal International 187 (3), 1301-1318.

Christensen, N., Mooney, W., 1995. Seismic velocity structure and composition of the continental crust; a global view. Journal of Geophysical Research-Solid Earth 100 (B6), 9761-9788.

Ebbing, J., Braitenberg, C., Götze, H., 2001. Forward and inverse modelling of gravity revealing insight into crustal structures of the Eastern Alps. Tectonophysics 337 (3-4), 191-208.

Fadel, I., Kerle, N., Meijde, M., 2015. 3D object oriented image analysis of geophysical data. Geophysical Journal International 35, 44-53.

Förste, C., Bruinsma, S., Flechtner, F., Marty, J.-C., Dahle, C., Abrykosov, O., Lemoine, J.-M., Neumayer, H., Barthelmes, F., Biancale, R., et al., 2013. Eigen-6c2-a new combined global gravity field model including GOCO data up to degree and order 1949 of GFZ-Potsdam and GRGS-Toulouse. In: EGU General Assembly Conference Abstracts, vol. 15, p. 4077.

Förste, C., Bruinsma, S., Shako, R., Marty, J., Flechtner, F., Abrikosov, O., Dahle, C., Lemoine, J., Neumayer, K., Biancale, R., et al., 2011. EIGEN-6-a new combined global gravity field model including goce data from the collaboration of GFZPotsdam and GRGS-Toulouse. In: Geophysical Research Abstracts, vol. 13.

Gardner, G., Gardner, L., Gregory, A., 1974. Formation velocity and density: the diagnostic basics for stratigraphic traps. Geophysics 39 (6), 770-780.

Godfrey, N., Beaudoin, B., Klemperer, S., 1997. Ophiolitic basement to the Great Valley forearc basin, California, from seismic and gravity data: implications for crustal growth at the North American continental margin. Geological Society of America Bulletin 109 (12), 1536-1562.

Götze, H., Lahmeyer, B., 1988. Application of three-dimensional interactive modeling in gravity and magnetics. Geophysics 53 (8), 1096-1108.

Haase, C., 2008. Improved estimation of subsurface magnetic properties using minimum mean-square error methods. Christian-Albrechts-University Kiel, Germany (Diploma thesis).

Korenaga, J., Holbrook, W.S., Detrick, R.S., Kelemen, P.B., 2001. Gravity anomalies and crustal structure at the southeast Greenland margin. Journal of Geophysical Research: Solid Earth 106 (B5), 8853-8870.

Liu, Q., Gu, Y., 2012. Seismic imaging: from classical to adjoint tomography. Tectonophysics 566-567, 31-66.

Martha, T., Kerle, N., van Westen, C., Jetten, V., Kumar, K., 2011. Segment optimization and data-driven thresholding for knowledge-based landslide detection by object-based image analysis. IEEE Transactions on Geoscience and Remote Sensing 49 (12), 4928-4943.

Mishra, D., Kumar, M.R., Arora, K., 2012. Long wavelength satellite gravity and geoid anomalies over Himalaya, and Tibet: lithospheric structures and seismotectonics of deep focus earthquakes of Hindu Kush Pamir and Burmese arc. Journal of Asian Earth Sciences 48, 93-110.

Ravat, D., Lu, Z., Braile, L., 1999. Velocity-density relationships and modeling the lithospheric density variations of the Kenya Rift. Tectonophysics 302 (3-4), 225-240.

Saether, B., 1997. Improved estimation of subsurface magnetic properties using minimum mean-square error methods. Norges teknisk-naturvitenskapelige univ, Trondheim, Norway (Ph.D. Thesis)

Sanchez, J., Götze, H., Schmitz, M., 2011. A 3-D lithospheric model of the CaribbeanSouth American plate boundary. International Journal of Earth Sciences 100 (7), 1697-1712.

Schmidt, S., Götze, H., Fichler, Ch.,A.M., 2010. IGMAS+: a new 3D gravity, FTG and magnetic modelling software. Extended abstract. Geoinformatik, 57-63.

Schmidt, S., Plonka, C., Götze, H., Lahmeyer, B., 2011. Hybrid modelling of gravity, gravity gradients and magnetic fields. Geophysical Prospecting 59 (6, SI), 1046-1051.

Schoenmeyer, R., Prvulovic, D., Rotarska-Jagiela, A., Haenschel, C., Linden, D.E.J., 2006. Automated segmentation of lateral ventricles from human and primate magnetic resonance images using cognition network technology. Magnetic Resonance Imaging 24 (10), 1377-1387.

Tugume, F., Nyblade, A., Juli, J., van der Meijde, M., 2013. Precambrian crustal structure in Africa and Arabia: evidence lacking for secular variation. Tectonophysics, http://dx.doi.org/10.1016/j.tecto.2013.04.027 (in press).

van der Meijde, M., Juli, J., Assumpo, M., 2013. Gravity derived Moho for South America. Tectonophysics, http://dx.doi.org/10.1016/j.tecto.2013.03.023 (in press).

van der Meijde, M., Pail, R., Bingham, R., Floberghagen, R., 2015. GOCE data, models, and applications; a review. Int. J. Appl. Earth Obs. Geoinform. 35, 4-15. 